action. The same thing will, of course, hold for a primary and the atmosphere of a satellite.

In the more general case, that in which the satellite has rotational velocity $\omega$ about its axis (supposed perpendicular to the plane of the orbit), we have, assuming that the satellite is spherical and denoting $\omega_{1}^{2}, / n^{2}$ by $c, m / \mathrm{M}>(2+c) a^{3} / r^{3}$. This agrees with the former result when $c=1$. These results were first obtained by M. Roche. ${ }^{1}$

The figure of a fluid satellite is determined by finding a surface to which the resultant of the gravitational pull of the primary on unit mass, a force $n^{2} r$ equal and opposite to the gravitational pull on unit mass at the centre, the gravitational force per unit mass exerted by the matter of the satellite itself, and the centrifugal force of unit mass, is everywhere perpendicular. A first approximation to the force due to the satellite itself is obtained by neglecting the deviation from sphericity, as is done above. But into this discussion we cannot here enter. It can only be stated that the final result, taking into account the distortion of the satellite, is that the satellite will be broken up if it approaches closer to the primary than the limit given by the inequality

$$
r>2 \cdot 44 \sqrt[3]{s_{1} / s_{1}} \text {. }
$$

Now imagine a planet and a satellite moving round the sun, the satellite being destitute of relative velocity. The satellite may, for example, be regarded as a particle (of unit mass say) of a ring of small mass composed of particles surrounding the primary at a distance $a$, the whole turning, if that were possible, with angular velocity equal to that of the primary round the sun. By what we have seen above, the excess of solar attraction over the sunward acceleration is, for a particle on the side nearest the sun, $3 k \mathrm{M} a / r^{3}$. This must be balanced by the attraction $\mathrm{km} / \mathrm{a}^{2}$, so that we have the equality $\mathrm{km} / \mathrm{a}^{2}=3 \mathrm{kM} a / \mathrm{r}^{3}$.

From these expressions for the forces we see that the potential energy, with a term for centrifugal force included, may be taken, for unit mass of the infinitesimal satellite on the line of centres at the point nearest the sun, as $-k m / a-\frac{3}{2} k \mathrm{M} a^{2} / r^{3}$. This is an example of the almost self-evident principle, known as the theorem of Coriolis, that if there be included a term in the potential energy which will give the components of centrifugal force, we may write down the equation of relative kinetic energy, just as if the rotating axes were fixed. The potential energy thus required for the centrifugal force on a particle of unit mass at a point at distances $\xi, \eta, \zeta$ from the planet's centre, measured respectively along the line of centres, perpendicular to this line in the plane of motion, and perpendicular to the plane of motion, is $-\frac{1}{2} n^{2}\left\{(r-\xi)^{2}+\eta^{2}\right\}$, or, since $n^{2} r=k \mathrm{M} / r^{2}$,

$$
-\frac{1}{2} k \mathrm{M}_{\{}\left\{(r-\xi)^{2}+\eta^{2}\right\} / r^{3} \text {. }
$$

The total potential energy being taken as

(with, as before,

$$
-\left[\frac{k m}{\rho}+\frac{k \mathrm{M}}{r_{I}}+\frac{\mathrm{I}}{2} \frac{k \mathrm{M}}{r^{3}}\left\{(r-\xi)^{2}+\eta^{2}\right\}\right]
$$

$$
v^{2}=\dot{\xi}^{2}+\dot{\eta}^{2}+\dot{\zeta}^{2}, \rho^{2}=\xi^{2}+\eta^{2}+\zeta^{2}, r_{1}^{2}=(r-\xi)^{2}+\eta^{2}+\zeta^{2},
$$

and $\left.n^{2}=k \mathrm{M} / r^{3}\right)$, the equation of relative kinetic energy is

$$
\frac{\mathrm{I}}{2} v^{2}-\left[\frac{k m}{\rho}+\frac{k \mathrm{M}}{r_{1}}+\frac{\mathrm{I}}{2} \frac{k \mathrm{M}}{r^{3}}\left\{(r-\xi)^{2}+\eta^{2}+\zeta^{2}\right\}\right]+\mathrm{C}=0,
$$

which, for an infinitesimal satellite, is Hill's equation as given above.

For the moon, which has mass $m^{\prime}$ sensible in comparison with the mass, $m$, of the earth, the first term in the square brackets should be $k\left(m+m^{\prime}\right) / \rho$.

It may be noticed by the dynamical student that if the above expression for the potential energy be denoted by $\mathrm{V}$, we have not $\ddot{\xi}=-\partial V / \partial \xi, \& c .$, for the equations of motion, but

$$
\xi-n \eta=-\partial \mathrm{V} / \partial \xi, \eta+n \xi=-\partial \mathrm{V} / \partial \eta \ddot{\zeta}=-\partial \mathrm{V} / \partial \zeta \text {. }
$$

A. Gray.

I I have found since the above was written that the same elementary view of this matter is given by Roche himself in his paper "Recherches sur les Atmosphères des Comètes," Annales de l'Observatoire, t v 850 Perhaps I may here direct attention to a valuable paper by Roche (which. Perhaps ever, though I have not seen it referred paproll oche (which may, how ever, though I have not seen it referred to, be well known to astronomers) de Montpellier, t, viii. This gives a gonic researches.

No. I6I 5, voL. 62 ]

\section{ANTELOPES AND THEIR RECOGNITION} $M A R K S$.

THE Tragelaphine Antelopes hold a unique position amongst the hollow-horned ruminants. No other group can show species so sharply contrasted in size and build as the massive eland rising over sixty inches at the withers. and the dainty little bush-buck which falls short of half that height. Only the Indian black buck amongst the gazelles can match the nylghaie and nyala for diversity of sexual colouring; and for elegance of form, coupled with beauty of marking and grandeur of carriage, the kudu is surpassed by no species of mammal.

Apart from certain features presented by the skull and horns, the affinity between the species here mentioned is attested by the markings of the skin. On a ground-colour shading from slate to chestnut are distributed certain white spots, patches or stripes, which crop up so persistently in the different genera as to leave no doubt they are a heritage from a common ancestor. A comparison between the skins of the existing species suggests that this ancestor was coloured somewhat as follows:-Body and head yellowish red ; flanks and hind-quarters striped with white; on the throat two white patches, one at each end; one or two spots on the cheeks, a V-shaped stripe between the eyes, a white chin, a white upper lip ; legs paler on the inner side, quite white at base close to chest and groin, and with two white spots on the pasterns in front.

Some or all of these markings have been inherited with scarcely an exception by every known species of Tragelaphine. Sometimes the spots on the head, sometimes the stripes on the body, sometimes the patches on the throat are suppressed; but even in extreme cases of suppression, a spot here, a stripe there, persists as a tell-tale sign of descent. The usefulness of characters so constant may be taken for granted. The nature of their usefulness has been discussed by both Wallace and Darwin; but so great is the discord between the opinions of these authorities that one cannot think both are right.

Referring to the importance of special marks for recognition where many species of nearly the same size and general form inhabit the same region, Mr. Wallace says : "It is interesting to note that these markings for recognition are very slightly developed in the antelopes of the woods and marshes. ... The wood-haunting bosch-bok ( $T$. sylvaticus) goes in pairs, and has hardly any distinctive markings on its dusky chestnut coat, but the male alone is horned. The large and handsome kudu frequents brushwood, and its vertical white stripes are no doubt protective, while its magnificent spiral horns afford easy recognition. The eland, which is an inhabitant of the open plain, is uniformly coloured, being sufficiently recognisable by its large size and distinctive form ; but the Derbyan eland is a forest animal, and has a protectively striped coat. In like manner, the fine Speke's antelope, which lives entirely in the swamps and among reeds, has pale vertical stripes on the sides (protective), with white markings on face and breast for recognition" ("Darwinism," p. 220).

It may be inferred from this passage that the interest attached to the slight development of recognition marks in the antelopes of the woods and marshes lies in the needlessness of such marks for species living apart and not herding with others of the same general size and form. If, however, there is no likelihood of confusion, it is not quite clear from what species the horns of the kudu serve to distinguish their owner, nor what significance in this connection is to be attributed to the occurrence of horns only in the male of the bosch-bok. Similarly, it is not clear what use Speke's marsh-buck can have for recognition marks. If, however, the spots on the face and throat subserve recognition in this species, we must also conclude they are retained for that purpose in the bong? ( $T$. euryceros), the lesser kudu, the nyala $(T$. angasi), in which they are very conspicuous, as well as in the various smaller kinds of bush-buck, which in other parts of Africa live the same life as the bosch-bok of the Colony. Surely, too, Derby's eland is at least as recognisable by its large size and distinctive form as the Cape species; yet it is adorned with a conspicuous V-shaped stripe between the eyes, and the lower throat-patch forms a white collar, standing boldly out against the black hue of the neck.

In short, if the marks in question have been preserved for recognition, it is singular that they are exceptionally well developed in the species that live in pairs or small parties by themselves in thick bush-species which, according to the hypothesis, have little, if any, need of them. It is conceded, of course, that the spots on the head and throat, like the stripes on the body, 
patches on the rump or any other visible external feature, including large ears, may, if required, serve as marks of identity ; but in the case of the Tragelaphines, at least, it is hard to believe that that is any more their primary function than it is the primary function of large ears.

A mass of evidence can be brought forward in favour of Wallace's view that the body-stripes of these antelopes are protective ; but there appear to me to be equally strong reasons for classifying the face and foot markings in the same category, and for regarding them as representing spots or streaks of sunlight passing through foliage or reflected from leaves.

It is possible, perhaps probable, that the other white patches on a typical Tragelaphine serve the same end; but their situation forcibly suggests that they have a still deeper significance. I believe they come within the scope of Thayer's hypothesis of concealment by the counteraction of light and shade. A convex body stands out amid surroundings of its own colour on account of the contrast between the light that is reflected from its upper surface and the shadow that pervades it below. Take away the light by darkening the upper side and the shadow by lightening the lower, and the body will vanish from sight with the destruction of its visible shape. By applying this principle to a typically marked Tragelaphine, the lesser kudu for example, it will be seen that the white is laid on where shadows are thrown; that the white rim on the upper lip and the white chin must counteract the shadows caused by the fold of the

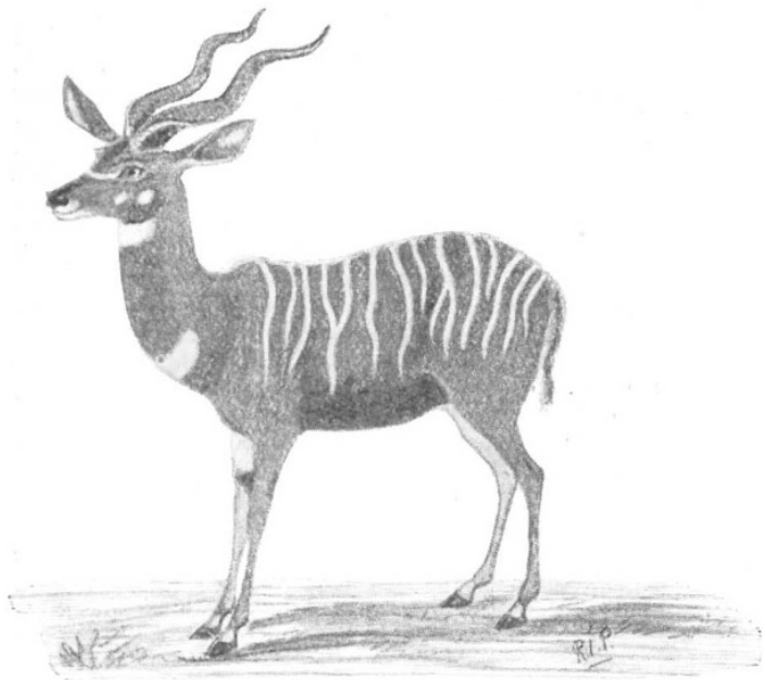

Frg. I.-Tre Lesser Kudu,

mouth and by the muzzle; the two white blotches on the neck must counteract the shadows thrown by the head and by the curvature of the throat, and the shadows cast by the breast and groin must be similarly obliterated by the white patches on the inner side of the base of the limbs.

That the white patches must have the effect here assigned to them will be obvious, I think, to any one who, with Thayer's hypothesis in mind, looks at a lesser kudu when it is standing full face. The reason for the presence of marks concealing the animal from this point of view will be referred to later on.

If the markings of the Tragelaphines have the significance here attached to them, they should be better developed in the species that live in the bush than in those that frequent the open. Let us see in a few cases to what extent they are correlated with habit. Two well-marked species of eland live in Africa, to wit, Derby's eland ( $T$. derbianus) from Senegambia and the commoner form $(T$.ory $x)$ which, with its subspecies, ranges throughout the whole of East and South Africa. The former, according to Winwood Reade, "lives in the forest, and never of its own accord enters the plain." It is reddish in colour, with a black neck, a white collar, eye-stripes and many white stripes on the flanks and hind-quarters. Of the common eland the typical "dun coloured" desert form is, according to Selous, "particularly plentiful in the dry desert country through which the Chobe runs," and examples "from the Kalahari desert have no sign of a stripe." Farther to the north, both in Angola, South east and East Africa, this unmarked type is replaced by its ancestral form, Livingstone's eland, which in colour and habits is intermediate between the Cape and Senegambian species. The skin is always marked with narrow white stripes, and the V-shaped mark between the eyes is often present. In British East Africa this eland, according to Jackson, was found in "sparsely timbered country and open bush bordering the plain rather than the plains themselves," and in Angola (Penrice) " it seems most partial to a thinly timbered country." There is thus a complete gradation from the strongly marked forest species through the weakly marked species frequenting the open bush to the unmarked desert species.

Take again the kudus. Both the species are well marked with white stripes on body and head, but the smaller (S. imberbis) is much more strikingly marked than the larger ( $S$. strepsiceros), having more stripes on the body and two patches on the throat. In Somaliland, where both species occur, the larger lives, according to Swayne, in the mountains, on very broken ground where there is plenty of bush; and sometimes indeed ventures into the open plain (Inverarity). The lesser kudu, on the contrary, "is found in thick jungles . . . especially where there is an undergrowth of the slender pointed aloe which grows from four to six feet high" (Swayne). Both Swayne and Inverarity, moreover, bear witness that this species will allow a hunter to get within a few yards before dashing away-a notorious habit with protectively coloured animals. Evidence of a like kind is furnished by other species of Tragelaphines. The beautifully marked nyala ( $T$. angasi) and bongo ( $T$. euryceros) live in dense thickets; and the lovely .little bush-bucks (T. sylvaticus, scriptus, \&c.) seldom venture out of cover except at night-time to feed. On the other hand, the nylghaie, an aberrant member of the same tribe, is without body-stripes, and lives for the most part in more or less open country in India, and is not a typical denizen of the thick jungle at all. ${ }^{1}$

Further evidence on this head is supplied by another set of facts. Ungulates which live in thickets or rough ground affording cover to enemies have larger ears and a keener sense of hearing than those of the plains or high mountains where intruders have little chance of concealment. Note the small ears of the camel, a typically desert form; or of goats and sheep which from the mountain peaks can sweep the surrounding country for miles with their eyes and seek safety in flight long before the foe gets within ear shot. Compare also the small equine ears of Burchell's zebra, which herds in the open plain free from obstacles to. interfere with vision, with the longer asinine ears of the mountain zebra which frequents rocky broken ground well fitted for the hiding of carnivores. In all the brilliantly marked Tragelaphines the ears are long and expanded, but in the nylghaie, and especially in the common eland, they are short and narrower. Indeed, one of the chief structural differences between Derby's eland and the Cape species is found in the size of the ears.

The co-existence of white marks with long ears and a bushlife bears out the supposition that the marks, like the ears, are primarily for protection, and that if subservient to purposes of recognition they are merely of secondary importance in that capacity.

I strongly suspect, too, that the markings of the sable, roan, gemsbok and bontebok are for concealment, and not for recogni. tion as $\mathrm{Mr}$. Wallace supposes. The theory of recornition marks as applied to these antelopes assu ues the need of some patch or spot to enable the members of a species to identify their own kind amongst the herds of other sorts living in the same place. The theory would rest upon a securer basis, if it could be shown that closely allied species feed together. But nothing, I suppose, is more certain than that, as a very general, perhaps invariable, rule, closely allied and similar species are not found together. If, for example, the gemsbok and the

${ }^{I}$ An apparent exception to the rule that the development of white stripes and spots is correlated with a jungle life is found in the Sitatunga and Speke's marsh-buck, which "live in vast reed beds and papyrus swamps, and only come into the open at night ". (Selous). Yet the stripes fade away in the adults of both sexes. Why is this? Possibly because these animals depend for concealment, not so much upon coloration that harmonises with that of the vegetation, as upon a newly acquired and efficient habit of biding under the water itself, with only the end of the nose jutting above the surface (Selous and Gedge).

2 Compare in this connection the small ears of the orang with the large ears of the chimpanzee. The former lives a more arboreal and therefore safer life than the latter, which requires quick hearing to enable it to escape to the tree; when feeding on the ground.

No. I6 5 , VOL. 62$]$ 
beisa lived side by side in the Kalahari, or Peters's palla and the common species in Rhodesia, there would perhaps be strong reasons for thinking that the differences in the facial bands, which enable us to recognise these species apart, serve the same end where the antelopes are themselves concerned. But the gemsbok and the beisa, the common and Angolan palla, never cross each other's path. Again, in cases where the geographical areas of two forms, closely allied, but distinguishable by bands or patches, meet, the two forms frequently interbreed, and so falsify the contention that marks keep like to like.

That ungulates of different sorts herd together is well known; we read, for example, of zebras, gnus, pallas, spring-boks and buffaloes feeding in each other's company on the veldt. But so distinct from each other in form are these animals and others that might occur with them, that it is rating their visual powers very low-much lower, indeed, than our own-to hold that they require special patches to keep them from committing the errors of identification which the hypothesis assumes they are liable to fall into. I believe, then, that the need for recognition marks in the case of antelopes has been much over-rated, 1 and is too slight to warrant the belief that the facial and other stripes of, say, the gemsbok or sable have been perfected by their usefulness as such. On the other hand, when we see that the pattern of the zebra is for concealment, that the network of white stripes on the giraffe blends with the lights passing

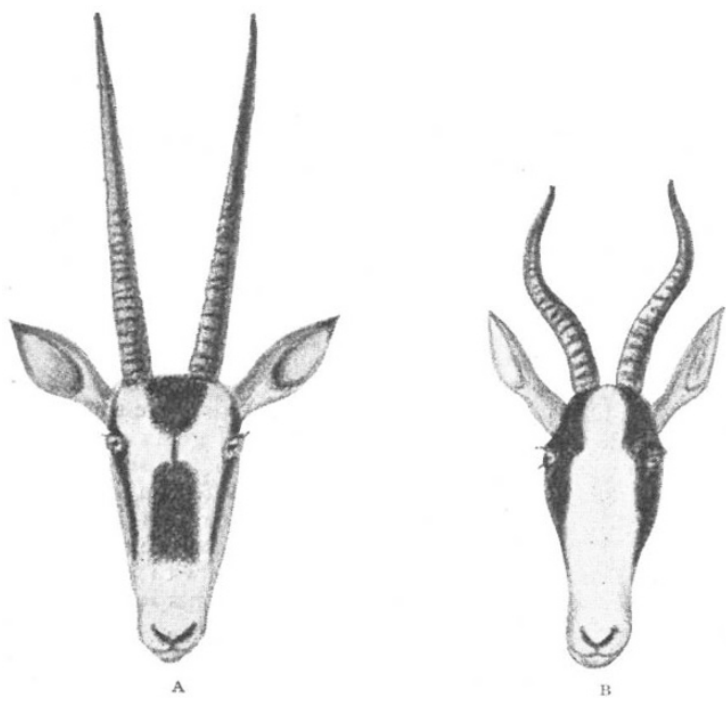

FIG. 2,-A, Face of the Beisa; B, face of the Bontebok.

through the intercrossing branches of trees, that the colour and shape of the feeding hartebeest are like those of the ant-hills, all these and other facts attesting the importance of concealment, we are justified in suspecting that the white blaze of the bontebok and the facial bands of the gazelles and oryxes are developed for concealment and not for recognition.

The markings take the form of strongly contrasted bands of white and black, or brown. Objects banded in this way are, as a rule, more, and not less, difficult to see in their natural surroundings than those that are uniformly coloured. There is little of the gloss on the coat of a grey or white horse that is seen on a bay or black, because white hair reflects the light less vividly than dark. Hence alternating bands of these hues im. part a blurred irregular aspect to a body, destroy the apparent evenness of its surface and break up the continuity of its outline. In an uncertain light a zebra's stripes " "merge into a grey tint," and mutually counteract each other, so that the animal is nearly invisible.

The stripes on the head of a gemsbok or sable are in a general way so like those on a zebra's coat that they must, one would

1 If the American prong-buck were an inhabitant of Africa, I presume that its conspicuous patterns, possibly indeed the unique shape of its horns, would be cited as evidence supporting the theory of recognition marks. But in the prairies of the United States there are no species that resemble it in size and form, so as to create confusion as to identity. The species it in size and form, so as to create confusion

$2 \mathrm{Mr}$. Wallace is surely "putting the cart before the horse" in the passage where he speaks of the coloration of the zebra as an instance of a style of marking for recognition becoming also protective.

NO. 1615 , VOL. 62$]$ think, have the effect of making the head inconspicuous. To explain the prevalence of such marks upon the head and forepart of the body, of which the quagga furnishes an illustration, the following suggestions may be made.

Once aware of an enemy's presence, an antelope has three chances of escape-concealment, flight and self-defence. Concealment is often the wisest course to pursue, especially where females and young are concerned. For concealment perfect stillness is of all things most important. Movement means detection, and detection may mean death. But it is necessary at the same time for every movement of the enemy to be scrutinised, so that the right moment for flying may be seized, when the necessity for flight becomes apparent. For this purpose the face must be turned towards the enemy and both eyes be kept upon him. In this watchful attitude little of the foreshortened body is visible from the enemy's point of view, and if the head of the antelope be carried low with the nape in a line with the spine, practically nothing of the animal is exposed but the head and the fore-legs. Hence the special importance of protective markings on these parts. ${ }^{1}$ Again, when lying on the ground the body will often be hidden by low scrub or, if cover be absent, may simulate a mound of earth or a termite's nest ${ }^{2}$; but the head, if protectively coloured, may with safety be raised to keep an eye on the surrounding country and guard against surprise.

The sexual colours of the Tragelaphines still remain to be touched upon. Darwin believed that the markings of the kudu, harnessed bush-buck, \&c., were in the first instance acquired by the male, then intensified by sexual selection, and partially transferred to the female. Against this hypothesis may be urged the evidence already adduced in favour of their protective ${ }^{3}$ value, and the distinctness they exhibit in the newlyborn or even fœetal young. Nevertheless a difference of colour, small or great, but as yet unexplained, does exist between the males and females of all the species of the group. It is noticeable, too, that the deviation affects the male; that it takes the direction of nigrescence, but by no means always of beauty, and that the female adheres to the typical coloration of the group.

There is no evidence, so far as I am aware, that the assumption of a dark coat by the male is connected with any peculiarity in mode of life of this sex, which would attest its use for concealment. On the contrary, since the colour-at least, in the case of the nylghaie ("Descent of Man," p. 535)-becomes intensified at the breeding season, without the growth of new hair, and has its appearance arrested by emasculation, its significance appears to be purely sexual and the outcome or accompaniment of "male katabolism." If so, it may have been intensified and fixed by the exercise of choice on the part of the females, or by the destruction or expulsion of their paler, less vigorous rivals by the stronger and darker males, which thus secured the females for themselves and left the greatest number of offspring.

It is significant that the three species of antelopes, namely the nyala, the nylghaie and the Indian black buck, in which the sexes differ strikingly from each other-differ indeed to an extent that is equalled by few species of mammals and surpassed by none--the female is without horns or other weapons of defence. This defencelessness, coupled with the exigencies of maternity, has compelled an adherence on her part to the normal protective coloration of the group; whereas the males, powerfully built and strongly horned, have been able to dispense to a great extent with colours that harmonise with those of the environment.

Warning characters are rare in mammals; but the difference in colour between the bull and cow of the species just mentioned may conceirably benefit the former at the expense sometimes of the latter by serving to distinguish him, the horned powerful fighter and dangerous antagonist when brought to bay, from her, the weak and defenceless one who may be attacked and pulled down with impunity. Why not? $\mathrm{R}$. I. Pocock.

I Presumably it is for an analogous reason that the tiger has a pair of sunlight patches on its face, so that its chances of concealment may be increased when watching for prey or creeping towards it.

2 In the case of the gemsbok, beis a and some gazelles the black longitudinal stripe passing along the side may enhance this resemblance by representing the shadow that is often seen where a boulder or mound meets the soil. The darkening of the coat on this area of the body, such as is seen, for instance, in the Asiatic wild ass (Kiang), should have the effect of deadening the light reflected from the bulging flank.

3 Here and elsewhere in this article I have purposely used the word

3 Here and elsewhere in this article I have purposely used the word familiar to general readers. 